\title{
Solving fuzzy differential equations via differential inclusions
}

\author{
Y. Chalco-Cano ${ }^{1}$ R. Rodríguez-López ${ }^{2}$ Geraldo N. Silva ${ }^{3}$ \\ ${ }^{1}$ Instituto de Alta Investigación, Universidad de Tarapacá, Casilla 7D, Arica, Chile \\ ${ }^{2}$ Dpto. Análisis Matemático, Universidad de Santiago de Compostela, Santiago de Compostela, 15782, Spain \\ ${ }^{3}$ Dpto. Mat. Aplicada, Instituto de Biociências, Letras e Ciências Exatas, UNESP, São José do Rio Preto-SP, Brasil
}

\begin{abstract}
In this paper we present a procedure to obtaining the solution of a fuzzy differential equation via differential inclusions. This procedure is an alternative approach, based on optimal control tools, to obtaining a description exact of a fuzzy solution for a large class of nonlinear fuzzy differential equations.
\end{abstract}

Keywords: Fuzzy differential equations, differential inclusions, optimal control

\section{Introduction}

Let us consider the following dynamic control system

$$
x^{\prime}=f(t, x, w), \quad x(0)=x_{0}, \quad w \in W \subset \mathbb{R}^{m}
$$

where $f:[0, T] \times \mathbb{R} \times \mathbb{R}^{m} \rightarrow \mathbb{R}$ is a continuous function. It is well-known that (1) is an important model for a wide class of real problems. However, in some cases, these equations are restrictive in their ability to describe phenomena. For example, in mathematical models that describe biological phenomena, the parameters and initial conditions usually are inherently uncertain and consequently the variables will also possess uncertainty, (see for example $[15,19])$. If we have a function whose parameters are fuzzy intervals in the right-hand side of (1), what is the appropriate extension within fuzzy theory?. To obtain a fuzzy differential equation from such a problem (1), we consider

$$
X^{\prime}(t)=\widehat{f}(t, X, U), \quad X(0)=X_{0} .
$$

where $\widehat{f}:[0, T] \times \mathcal{F}_{C} \times \mathcal{F}_{C}^{m} \rightarrow \mathcal{F}_{C}^{n}$ was obtained from a continuous function $f:[0, T] \times \mathbb{R} \times \mathbb{R}^{m} \rightarrow \mathbb{R}$ by applying Zadeh's extension principle, $X_{0} \in \mathcal{F}_{C}$ and $U \in \mathcal{F}_{C}^{m}(W)$.

For problem (2) there are at least three possibilities for representing a fuzzy solution: the first involves the derivative of fuzzy functions $[3,4,8,9,10$, $24]$; the second is obtained by applying Zadeh's extension principle to the deterministic solution $[9,20]$ and the last one is based on a family of differential inclusions $[2,7,12,13,16,17,18]$. In this article we devote to the last approach, obtaining a fuzzy solution of (2) via a family of differential inclusions. In particular we present a procedure for obtaining a solution of (2) based on optimal control tools. With this procedure we obtain a description exact of a solution for a large class of nonlinear fuzzy differential equations.

\section{Notation, the space of fuzzy sets and fuzzy arithmetic}

On the space of all compact and convex intervals $\mathcal{K}_{C}$, we have the standard interval arithmetic which is due Moore [21]. He states that, given two intervals $A$ and $B$,

$A * B=\{c \mid c=a * b, a \in A, b \in B, * \in\{+,-, \times, \div\}\}$,

and the multiplication of a real number $\lambda$ by an interval $A=[\underline{a}, \bar{a}]$ is given by

$$
\lambda A=[\min \{\lambda \underline{a}, \lambda \bar{a}\}, \max \{\lambda \underline{a}, \lambda \bar{a}\}] .
$$

Recall that a fuzzy set $u$ on a universe set $X$ is a mapping $u: X \rightarrow[0,1]$. We think of $u$ as assigning to each element $x \in X$ a degree of membership, $0 \leq u(x) \leq 1$. If $u$ is a fuzzy set on $\mathbb{R}^{n}$, we define $[u]^{\alpha}=\left\{x \in \mathbb{R}^{n} \mid u(x) \geq \alpha\right\}$ the $\alpha$-level of $u$, with $0<\alpha \leq 1$. For $\alpha=0$ the support of $u$ is defined as $[u]^{0}=\operatorname{supp}(u)=\overline{\left\{x \in \mathbb{R}^{n} \mid u(x)>0\right\}}$, where $\bar{A}$ denotes the closure of $A \subset \mathbb{R}^{n}$.

We denote by $\mathcal{F}_{C}$ the family of all fuzzy intervals, i.e. the family of all fuzzy sets such that $\alpha$-level sets $[u]^{\alpha}$ are nonempty compact intervals. We denote by $[u]^{\alpha}=\left[\underline{u}_{\alpha}, \bar{u}_{\alpha}\right]$ the $\alpha$-level of a fuzzy interval $u$. If the core of a fuzzy interval $u$ is a singleton, i.e. if $[u]^{1}=\{a\}$, with $a \in \mathbb{R}$, then $u$ is called fuzzy number. A triangular fuzzy number $u$ is fuzzy interval which is well-defined by three real numbers: $u=(a, b, c)$ with $a, b, c \in \mathbb{R}$ and $a \leq b \leq c$ where $[a, c]$ is the support and $b$ is the core of $u$.

The following characterization of elements of $\mathcal{F}_{C}$ is important in fuzzy theory.

Theorem 1 Let $\left\{Y_{\alpha} \subset \mathbb{R} \mid 0 \leq \alpha \leq 1\right\}$ be a family of sets satisfying the following:

- $Y_{\alpha}$ is a compact and convex interval, for all $0 \leq \alpha \leq 1$;

- $Y_{\beta} \subset Y_{\alpha}$ for $0 \leq \alpha \leq \beta \leq 1$;

- $Y_{\alpha}=\cap_{i=1} Y_{\alpha_{i}}$ for any nondecreasing sequence $\alpha_{i} \rightarrow \alpha$ in $[0,1]$.

Then there is a unique fuzzy interval $u \in \mathcal{F}_{C}$ such that $[u]^{\alpha}=Y_{\alpha}$. Conversely, the level sets $[u]^{\alpha}$ for any $u \in \mathcal{F}_{C}$ satisfy these conditions. 
Given two fuzzy intervals $u_{1}$ and $u_{2}$ we can define the pair $\left(u_{1}, u_{2}\right)$, a fuzzy set on $\mathbb{R}^{2}$, such that

$$
\left(u_{1}, u_{2}\right)(x, y)=u_{1}(x) \wedge u_{2}(y),
$$

where $\wedge$ denotes the minimum. Then $\left[\left(u_{1}, u_{2}\right)\right]^{\alpha}=$ $\left[u_{1}\right]^{\alpha} \times\left[u_{2}\right]^{\alpha}$, for all $\alpha \in[0,1]$, where $\times$ denotes the usual Cartesian product [11]. So, we can define a fuzzy vector as being $\left(u_{1}, \ldots, u_{n}\right)$ such that $u_{i} \in \mathcal{F}_{C}$, for $i=1, \ldots, n$. We denote by $\left(\mathcal{F}_{C}\right)^{n}$ the space of all fuzzy vectors, that is,

$$
\left(\mathcal{F}_{C}\right)^{n}=\left\{\left(u_{1}, \ldots, u_{n}\right) \mid u_{i} \in \mathcal{F}_{C}, i=1, \ldots, n\right\} .
$$

We can extend the Hausdorff-Pompieu metric $H$ on $\mathcal{K}^{n}$ to $\mathcal{F}^{n}$ by means

$$
D(u, v)=\sup _{\alpha \in[0,1]} H\left([u]^{\alpha},[v]^{\alpha}\right), \forall u, v \in \mathcal{F}_{C}^{n} .
$$

It is well-known that $\left(\mathcal{F}^{n}, D\right)$ is a complete metric space and $\left(\mathcal{F}_{C}^{n}, D\right)$ is a closed subspace of $\left(\mathcal{F}^{n}, D\right)$.

A function $f: \mathbb{R}^{m} \rightarrow \mathbb{R}^{n}$ induces a unique fuzzy function $\hat{f}:\left(\mathcal{F}_{C}\right)^{m} \rightarrow\left(\mathcal{F}_{C}\right)^{n}$ defined by

$$
\hat{f}(u)(y)=\left\{\begin{array}{lll}
\sup _{x \in \mathbb{R}^{m}, f(x)=y} u(x) & \text { if } & f^{-1}(y) \neq \emptyset \\
0 & \text { if } & f^{-1}(y)=\emptyset .
\end{array}\right.
$$

We say that $\hat{f}$ is obtained from $f$ by applying the Zadeh's extension principle (ZPE).

Arithmetic operations are continuous real-valued functions, excluding division by zero. Therefore, the extension principle can be used for obtaining a fuzzy arithmetic [11]. For instance, if we consider the function $f_{+}: \mathbb{R}^{2} \rightarrow \mathbb{R}$ defined by $f_{+}(x, y)=x+$ $y$, applying ZPE we obtain a unique fuzzy function $\hat{f}_{+}:\left(\mathcal{F}_{C}\right)^{2} \rightarrow \mathcal{F}_{C}$ defined by

$$
\hat{f_{+}}(u, v)(x)=\sup _{x_{1}+x_{2}=x} u\left(x_{1}\right) \wedge v\left(x_{2}\right) .
$$

Thus, we define the sum between two fuzzy intervals $u, v \in \mathcal{F}_{C}$ by $u+v=\hat{f}_{+}(u, v)$. Also, considering the function $f_{m}$ defined by $f_{m}(x)=\lambda \cdot x$, with $\lambda \in \mathbb{R}$, and applying ZPE we obtain $\lambda \cdot u=\widehat{f_{m}}(u)$, the multiplication of a scalar $\lambda$ by a fuzzy interval $u$.

Any continuous function $f$ can be extend to a unique fuzzy function by applying the extension principle. For example, if we consider the function $f_{1}: \mathbb{R}^{+} \rightarrow \mathbb{R}$ defined by $f_{1}(x)=\sqrt{x}$, applying ZPE we obtain the fuzzy function $\hat{f}_{1}: \mathcal{F}_{C}\left(\mathbb{R}^{+}\right) \rightarrow \mathcal{F}_{C}$ and we define $\sqrt{u}$ by

$$
\sqrt{u}=\hat{f}_{1}(u)
$$

for all fuzzy interval $u$ such that $[u]^{0} \subset \mathbb{R}^{+}$.

In general, the computation of the fuzzy function $\hat{f}$ is a complex problem. But there exists a relation between the $\alpha$-levels of $\hat{f}(u)$ and the image of $\alpha$-level of $u$ by $f$, which helps to obtain $\hat{f}$.

Theorem 2 ([25]) The function $f: \mathbb{R}^{n} \rightarrow \mathbb{R}^{m}$ is continuous if and only if its extension $\hat{f}:\left(\mathcal{F}_{C}\right)^{n} \rightarrow$
$\left(\mathcal{F}_{C}\right)^{m}$ is a well-defined function and it is continuous with respect to the Haussdorff-Pompieu metric. Moreover

$$
\left[\hat{f}\left(u_{1}, \ldots, u_{n}\right)\right]^{\alpha}=f\left(\left[u_{1}\right]^{\alpha}, \ldots,\left[u_{n}\right]^{\alpha}\right),
$$

for all $\alpha \in[0,1]$ and for all $u_{i} \in \mathcal{F}_{C}$, where $f(A)=$ $\{f(a) / a \in A\}$.

Theorem 2 and equation (8) states that we can obtain the sum of two fuzzy intervals $u+v$ applying ZPE (6) and

$$
[u+v]^{\alpha}=[u]^{\alpha}+[v]^{\alpha}, \quad \forall \alpha \in[0,1],
$$

where $[u]^{\alpha}+[v]^{\alpha}$ is the sum of two compact intervals defined by (3). Multiplication of a fuzzy set by a scalar $\lambda \cdot u$ has the following property:

$$
[\lambda \cdot u]^{\alpha}=\lambda[u]^{\alpha}, \quad \forall \alpha \in[0,1]
$$

where $\lambda[u]^{\alpha}$ is the multiplication of a scalar by a compact interval (4).

In addition to the sum and multiplication by a scalar, subtraction $u-v$, multiplication $u \times v$ and division $u \div v\left(0 \notin[v]^{0}\right)$ between two fuzzy intervals $u, v$ can be obtained by applying ZPE. For more details see [7].

\section{Fuzzy differential equations via differential inclusions}

We consider the following class of fuzzy differential equations

$$
X^{\prime}(t)=U_{1}(t) \cdot \hat{g}(t, X(t))+U_{2}(t), \quad X(0)=X_{0} .
$$

where $X_{0}, U_{1}(t), U_{2}(t) \in \mathcal{F}_{C}$ and $\hat{g}:[0, T] \times \mathcal{F}_{C} \rightarrow$ $\mathcal{F}_{C}$ is obtained from a real-valued continuous function $g:[0, T] \times \mathbb{R} \rightarrow \mathbb{R}$ by applying the Zadeh's extension principle.

We assume that $g$ is any function which is continuous in the first argument and continuously differentiable in the second argument. Observe that the system above is not necessarily linear, it may be nonlinear in $x$. If $g(t, x)=x$ the fuzzy differential equation (9) is certainly a linear one. Also, we assume that $g(t, x) \neq 0$ for almost all $(t, x)$ when $U_{2}=0$.

The fuzzy initial value problem (9) can be rewritten as a family of differential inclusions (see [7])

$$
x_{\alpha}^{\prime}(t) \in\left[U_{1}(t)\right]^{\alpha} \cdot g\left(t, x_{\alpha}(t)\right)+\left[U_{2}(t)\right]^{\alpha}, \quad x_{\alpha}(0) \in\left[X_{0}\right]^{\alpha},
$$

with $0 \leq \alpha \leq 1$. The subscript $\alpha$ indicates the $\alpha$-level of the problem, i.e we have a differential inclusion at each $\alpha$-level.

We denote by $\mathcal{A}_{\alpha}\left(t, X_{0}^{\alpha},[U]^{\alpha}\right)$, where $U=U_{1} \times$ $U_{2}$, the attainable sets associated with problem (10) and it is defined, for each $\alpha \in[0,1]$, by

$$
\begin{aligned}
& \mathcal{A}_{\alpha}\left(t, X_{0}^{\alpha},[U]^{\alpha}\right) \\
= & \left\{x_{\alpha}(t) / x_{\alpha}(\cdot) \text { is a solution of }(10) \text { in }[0, T]\right\} .
\end{aligned}
$$


For each $t \in[0, T]$ the family $\left\{\mathcal{A}_{\alpha}\left(t, X_{0}^{\alpha},[U]^{\alpha}\right)\right\}_{\alpha \in[0,1]}$ satisfies the conditions of the Theorem 1 (see $[1,7,12]$ ) and so there exists an interval fuzzy $\mathcal{A}\left(t, X_{0}, U\right)$ which become a fuzzy solution $X(t)=\mathcal{A}\left(t, X_{0}, U\right)$ of (9) via differential inclusion and

$$
\left[\mathcal{A}\left(t, X_{0}, U\right)\right]^{\alpha}=\mathcal{A}_{\alpha}\left(t,\left[X_{0}\right]^{\alpha},[U]^{\alpha}\right), \quad \forall \alpha \in[0,1] .
$$

From above, we can see that obtaining a solution of the fuzzy differential equation (9) is equivalent to finding the attainable sets of the family of differential inclusions (10).

How do we obtain $\mathcal{A}_{\alpha}\left(t, X_{0}^{\alpha},[U]^{\alpha}\right)$ for each $\alpha \in$ $[0,1]$ ?. It is well-known that the family of differential inclusions (10) is equivalent to a family of dynamical systems controlled by parameters $c_{\alpha}(t) \in$ $[U]^{\alpha}$ (for more details see $[5,7]$ )

$x_{\alpha}^{\prime}(t)=c_{1, \alpha}(t) \cdot x_{\alpha}(t)+c_{2, \alpha}(t), x_{\alpha}(0)=x_{0}^{\alpha} \in\left[X_{0}\right]^{\alpha}$,

where $c_{\alpha}=\left(c_{1, \alpha}(t), c_{2, \alpha}(t)\right) \in\left[U_{1}\right]^{\alpha} \times\left[U_{2}\right]^{\alpha}=[U]^{\alpha}$.

Let us denote by $\mathcal{M}\left([0, T],[U]^{\alpha}\right)$ the family of all the measurable functions $c_{\alpha}:[0, T] \rightarrow \mathbb{R}^{2}$ such that $c_{\alpha}(t) \in[U]^{\alpha}$. So, for each $c_{\alpha} \in \mathcal{M}\left([0, T],[U]^{\alpha}\right)$ and $x_{0}^{\alpha} \in\left[X_{0}\right]^{\alpha}$ we have a unique solution $x_{\alpha}\left(t, c_{\alpha}, x_{0}^{\alpha}\right)$ of the problem (11) and it is also a solution of the differential inclusion (10) [5, 27]. Thus, for obtaining $\mathcal{A}_{\alpha}\left(t, X_{0}^{\alpha},[U]^{\alpha}\right)$, with $\alpha \in[0,1]$, we need to consider all the measurable functions $c_{\alpha} \in$ $\mathcal{M}\left([0, T],[U]^{\alpha}\right)$, all initial conditions $x_{0}^{\alpha} \in\left[X_{0}\right]^{\alpha}$ and, for each one of these, obtain the solutions $x_{\alpha}\left(t, c_{\alpha}, x_{0}^{\alpha}\right)$ associated with $c_{\alpha}$ and $x_{0}^{\alpha}$. Thus,

$$
\begin{aligned}
& \mathcal{A}_{\alpha}\left(t, X_{0}^{\alpha},[U]^{\alpha}\right)= \\
& \bigcup x_{\alpha}\left(t, c_{\alpha}, x_{0}^{\alpha}\right), \\
& c_{\alpha}(\cdot) \in \mathcal{M}\left([0, T],[U]^{\alpha}\right) \\
& x_{0}^{\alpha} \in\left[X_{0}\right]^{\alpha}
\end{aligned}
$$

for each $\alpha \in[0,1]$.

Next we present a procedure to finding a fuzzy solution of (9). Let us denote $\left[X_{0}\right]^{\alpha}=\left[\underline{x}_{0} \alpha, \overline{x_{0}} \alpha\right]$, $\left[U_{i}\right]^{\alpha}=\left[u_{i}, \bar{u}_{i}\right], i=1,2$.

For each $\alpha \in[0,1]$ we consider the following optimal control problems:

$$
\begin{array}{ll}
\min & x_{\alpha}(T) \\
\text { s.a. } & x_{\alpha}^{\prime}=c_{1, \alpha} \cdot g\left(t, x_{\alpha}\right)+c_{2, \alpha} \\
& \frac{u_{1}}{u_{\alpha}}(t) \leq c_{1, \alpha}(t) \leq \overline{u_{1}} \alpha(t), t \in[0, T] \\
& \frac{u_{2} \alpha}{x(0) \in c_{2, \alpha}(t) \leq \bar{u}_{2} \alpha}(t), t \in[0, T] \\
& x\left(\underline{x}_{0}, \bar{x}_{0} \alpha\right] .
\end{array}
$$

and

$$
\begin{array}{ll}
\max & x_{\alpha}(T) \\
\text { s.a. } & x_{\alpha}^{\prime}=c_{1, \alpha} \cdot g\left(t, x_{\alpha}\right)+c_{2, \alpha} \\
& \frac{u_{1}}{u_{2}}(t) \leq c_{1, \alpha}(t) \leq \bar{u}_{1} \alpha(t), t \in[0, T] \\
& \left.\frac{u_{2}}{x(0) \in\left[\underline{x}_{0}, \alpha\right.}, \bar{x}_{0} \alpha\right]
\end{array}
$$

Lemma 1 Under the above assumptions, for each $t \in[0, T]$, the fuzzy solution $X$ of (9) is given by

$$
[X(t)]^{\alpha}=\mathcal{A}_{\alpha}\left(t, X_{0}^{\alpha},[U]^{\alpha}\right)=\left[\underline{x}_{\alpha}^{*}(t), \bar{x}_{\alpha}^{*}(t)\right],
$$

where $\underline{x}_{\alpha}^{*}(t)$ and $\bar{x}_{\alpha}^{*}(t)$ are optimal solutions of (13) and (14), respectively.

Proof One of the properties of attainable sets is the fact that if a trajectory reaches a point on the boundary of the attainable set at the final time, then its entire graph must belong to the attainable boundary, for details see $[14,22,27]$. Based on this property we get (15).

From previous lemma we obtain the fuzzy solution of (9) solving the optimal control problems (13) and (14). Indeed, let us apply the maximum principle to (13) and (14) (see, for example, [29]). For this, we denote by $\underline{x}_{\alpha}^{*}$ and $\bar{x}_{\alpha}^{*}$ the corresponding optimal trajectory. So we obtain $\underline{x}_{\alpha}^{*}(0)=x_{0}$ and $\bar{x}_{\alpha}^{*}(0)=\bar{x}_{0}$, and fix $t \in[0, T]$ solving (13) we obtain that it is equivalent to solving the following optimization problem (see [6])

$$
\left.\begin{array}{cl}
\min & c_{1, \alpha} \cdot g\left(t, \underline{x}_{\alpha}^{*}(t)\right)+c_{2, \alpha} \\
\mathrm{s.t.} & \frac{u_{1}}{} \alpha(t) \leq c_{1, \alpha} \leq \bar{u}_{1} \alpha \\
& {\underline{u_{2}}}_{\alpha}(t) \leq c_{2, \alpha} \leq \bar{u}_{2} \alpha \\
& (t) .
\end{array}\right\}
$$

In the same way, we obtain that solving (14) is equivalent to solving the following optimization problem

$$
\left.\begin{array}{cl}
\max & c_{1, \alpha} \cdot g\left(t, \bar{x}_{\alpha}^{*}(t)\right)+c_{2, \alpha} \\
\text { s.t. } & \frac{u_{1}}{u_{\alpha}}(t) \leq c_{1, \alpha} \leq \overline{u_{1}} \alpha(t) \\
& {\underline{u_{2}}}_{\alpha}(t) \leq c_{2, \alpha} \leq \bar{u}_{2} \alpha(t) .
\end{array}\right\}
$$

From above we have the following result.

Theorem 3 For each $t \in[0, T]$, the fuzzy solution $X$ of (9) at time $t$ is such that $[X(t)]^{\alpha}=$ $\left[\underline{x}_{\alpha}^{*}(t), \bar{x}_{\alpha}^{*}(t)\right]$, where, for each $t \in[0, T]$,

$\underline{x}_{\alpha}^{*}(t)=\min \left\{x_{\alpha, 1}\left(\underline{x}_{0}, t\right), x_{\alpha, 2}\left(\underline{x}_{0}, t\right), x_{\alpha, 3}\left(\underline{x}_{0}, t\right), x_{\alpha, 4}\left(\underline{x}_{0}, t\right)\right\}$,

$\bar{x}_{\alpha}^{*}(t)=\max \left\{x_{\alpha, 1}\left(\bar{x}_{0}, t\right), x_{\alpha, 2}\left(\bar{x}_{0}, t\right), x_{\alpha, 3}\left(\bar{x}_{0}, t\right), x_{\alpha, 4}\left(\bar{x}_{0}, t\right)\right\}$.

Here $x_{\alpha, i}\left(\underline{x}_{0}, t\right)$ and $x_{\alpha, i}\left(\bar{x}_{0}, t\right)$ are the solutions of the equations

$$
\left\{\begin{array}{l}
x_{\alpha, 1}^{\prime}=\bar{u}_{1} \alpha(t) g\left(t, x_{\alpha, 1}(t)\right)+u_{2} \alpha(t), \\
x_{\alpha, 2}^{\prime}=\bar{u}_{1} \alpha(t) g\left(t, x_{\alpha, 2}(t)\right)+\overline{\bar{u}}_{2} \alpha(t), \\
x_{\alpha, 3}^{\prime}=\overline{\bar{u}}_{\alpha}(t) g\left(t, x_{\alpha, 3}(t)\right)+u_{2}(t), \\
x_{\alpha, 4}^{\prime}=\overline{u_{2}} \alpha(t) g\left(t, x_{\alpha, 4}(t)\right)+\overline{\bar{u}}_{2} \alpha(t),
\end{array}\right.
$$

with initial conditions $x_{\alpha, i}(0)=\underline{x}_{0}$ and $x_{\alpha, i}(0)=$ $\bar{x}_{0} \alpha$, for $i=1,2,3,4$, respectively.

Proof Fixed $\alpha \in[0,1]$, necessary conditions of optimality applied to the optimization problem (16) implies that

$\nabla\left[c_{1, \alpha} \cdot g\left(t, \underline{x}_{\alpha}^{*}(t)\right)+c_{2, \alpha}\right]=\left(g\left(t, \underline{x}_{\alpha}^{*}(t)\right), 1\right) \neq(0,0)$,

for all $c_{1, \alpha} \in\left[\underline{u}_{1}(t), \overline{u_{1}} \alpha(t)\right], c_{2, \alpha} \in\left[\underline{u}_{2} \alpha(t), \overline{u_{2}} \alpha(t)\right]$. Furthermore, provided the set of constraints is a simplex and the problem is linear in $\left(c_{1, \alpha}, c_{2, \alpha}\right)^{1}$, we know that the optimal solutions are extreme points of the simplex. Thus, for each $t \in[0, T]$,

\footnotetext{
${ }^{1}$ Observe that $t$ is fixed and $g$ is being evaluated at $\underline{x \alpha}^{*}(t)$
} 
either $\left(c_{1, \alpha}^{*}, c_{2, \alpha}^{*}\right)=\left(u_{1_{\alpha}}(t), u_{2}(t)\right)$ or $\left(c_{1, \alpha}^{*}, c_{2, \alpha}^{*}\right)=$ $\left(\underline{u}_{1} \alpha(t), \bar{u}_{2} \alpha(t)\right)$ or $\left(\bar{c}_{1, \alpha}^{*}, c_{2, \alpha}^{*}\right)=\left(\bar{u}_{1}(t), \underline{u}_{2}(t)\right)$ or $\left(\overline{c_{1, \alpha}^{*}}, c_{2, \alpha}^{*}\right)=\left(\bar{u}_{1} \alpha(t), \bar{u}_{2} \alpha(t)\right)$.

We can now conclude that the lower bound $\underline{x}_{\alpha}^{*}(t)$ of the set $[X(t)]^{\alpha}$ is obtained by setting

$\underline{x}_{\alpha}^{*}(t)=\min \left\{x_{\alpha, 1}\left(\underline{x}_{0}, t\right), x_{\alpha, 2}\left(\underline{x}_{0}, t\right), x_{\alpha, 3}\left(\underline{x}_{0}, t\right), x_{\alpha, 4}\left(\underline{x}_{0}, t\right)\right\}$

where $x_{\alpha, i}\left(\underline{x}_{0}, t\right)$ is the solution of the $i$-th equation in (18) with $x_{\alpha, i}(0)=x_{0}, i=1,2,3,4$.

Similar reasoning, making the appropriate changes, allows us to conclude that

$\bar{x}_{\alpha}^{*}(t)=\min \left\{x_{\alpha, 1}\left(\bar{x}_{0}, t\right), x_{\alpha, 2}\left(\bar{x}_{0}, t\right), x_{\alpha, 3}\left(\bar{x}_{0}, t\right), x_{\alpha, 4}\left(\bar{x}_{0}, t\right)\right\}$

where $x_{\alpha, i}\left(\bar{x}_{0}, t\right)$ is the solution of the $i$-th equation in (18) with $x_{\alpha, i}(0)=\underline{x}_{0}, i=1,2,3,4$. The theorem is proved. $\square$

As a consequence of the Theorem 3 we have the following result for linear case i.e. for the following linear fuzzy differential equation

$$
X^{\prime}=a(t) X+b(t) W, \quad X(0)=X_{0},
$$

where $a, b:[0, T] \rightarrow \mathbb{R}$ are continuous functions and $W, X_{0} \in \mathcal{F}_{C}$, where $[W]^{\alpha}=\left[\underline{w}_{\alpha}, \bar{w}_{\alpha}\right]$ and $\left[X_{0}\right]^{\alpha}=$ $\left[\underline{x}_{0} \alpha, \bar{x}_{0}\right]$.

Corollary 1 Let $X$ be the fuzzy solution of (20) then the extreme functions $\underline{x}_{\alpha}^{*}$ and $\bar{x}_{\alpha}^{*}$ are such that: (i) $\underline{x}_{\alpha}^{*}$ is solution of the ordinary differential equation

$x^{\prime}(t)=a(t) x(t)+\left(\frac{\bar{w}_{\alpha}+\underline{w}_{\alpha}}{2}\right) b(t)-\left(\frac{\bar{w}_{\alpha}-\underline{w}_{\alpha}}{2}\right)|b(t)|$,

with $x(0)=x_{0} \alpha$;

(ii) $\bar{x}_{\alpha}^{*}$ is solution of the ordinary differential equation

$x^{\prime}(t)=a(t) x(t)+\left(\frac{\bar{w}_{\alpha}+\underline{w}_{\alpha}}{2}\right) b(t)+\left(\frac{\bar{w}_{\alpha}-\underline{w}_{\alpha}}{2}\right)|b(t)|$,

with $x(0)=\bar{x}_{0} \alpha$.

The previous Corollary 1 was obtained in [7] where we also can find some examples to applying the result.

\section{Acknowledgments.}

This paper has been supported by Fondecyt-Chile through project 1151154, by FAPESP-Brazil under grants (11/01977-2, 11/13985-0, and 2011/51305$0)$ and by CNPq under grant 402633/2012-1, and by Ministerio de Economía y Competitividad, projects MTM2010-15314 and MTM2013-43014P, co-financed by the European Community fund FEDER.

\section{References}

[1] J.P. Aubin, A. Cellina, Differential Inclusions, Springer-Verlag, New York, 1984.
[2] V. A. Baidosov, Fuzzy differential inclusions, Journal of Applied Mathematics and Mechanics, 54:8-13, 1990.

[3] B. Bede and S. G. Gal, Generalizations of the differentiability of fuzzy number valued functions with applications to fuzzy differential equation, Fuzzy Sets and Systems, 151:581-599, 2005.

[4] B. Bede and S.G. Gal, Solutions of fuzzy differential equations based on generalized differentiability, Communications in Mathematical Analysis 9 (2010) 22-41.

[5] V.I. Blagodatskikh, A.F. Filippov, Differential inclusions and optimal control, Proceedings of the Steklov Institute of Mathematics 169 (1986) 199-259.

[6] Y. Chalco-Cano, V.A. de Oliveira, G.N. Silva, Description of the Attainable Sets of OneDimensional Differential Inclusions, J. Optim. Theory Appl., DOI 10.1007/s10957-014-0563-1.

[7] Y. Chalco-Cano, H. Román-Flores, Some remark on fuzzy differential equations via differential inclusions, Fuzzy Sets and Systems 230 (2013) 3-20.

[8] Y. Chalco-Cano, H. Román-Flores, On new solution of fuzzy differential equations, Chaos, Solitons \& Fractals 38 (2008) 112-119.

[9] Y. Chalco-Cano, H. Román-Flores, Comparation between some approaches to solve fuzzy differential equations, Fuzzy Set and Systems 160 (2009) 1517-1527.

[10] Y. Chalco-Cano, W.A. Lodwick, B.Bede, Fuzzy differential equations and Zadeh's extension principle, Proceedings of 30th NAFIPS International Conference IEEE, El Paso 2011.

[11] P. Diamond and P.E. Kloeden, Metric Spaces of Fuzzy Sets: theory and applications, World Scientific, Singapore, 1994.

[12] P. Diamond, Time-dependent differential inclusions, cocycle attractors and fuzzy differential equations, IEEE Trans. Fuzzy Systems 7 (1999) 734-740.

[13] P. Diamond, Stability and periodicity in fuzzy differential equations, IEEE Trans. Fuzzy Systems 8 (2000) 583-590.

[14] H. Frankowska, Contingent cones to reachable sets of control systems, SIAM Journal of Control and Optimization 27 (1989) 170-198.

[15] M. Guo, X. Xue, R. Li, Impulsive functional differential inclusions and fuzzy population models, Fuzzy Sets and Systems 138 (2003) 601-615.

[16] E. Hüllermeier, An approach to modeling and simulation of uncertain dynamical systems, International Journal Uncertainty, Fuzziness Knowledge-Based Systems 5 (1997) 117137.

[17] E. Hüllermeier, Numerical methods for fuzzy initial value problems, International Journal Uncertainty, Fuzziness Knowledge-Based Systems 7 
(1999) 1-23.

[18] V. Lakshmikantham, R.N. Mohapatra, Theory of Fuzzy Differential Equations and Inlusions, Taylor \& Francis, New York, 2003.

[19] K.K. Majumdar, D.D. Majumder, Fuzzy differential inclusions in atmospheric and medical cybernetics, IEEE Transaction on Systems, and Cybernetics - Part B: Cybernetics 34 (2004) 877887.

[20] M. Misukoshi, L.C. Barros, Y. Chalco-Cano, H. Román-Flores, and R.C. Bassanezi, Fuzzy differential equations and the extension principle, Information Sciences 177 (2007) 3627-3635.

[21] R.E. Moore, Interval Analysis, Prince-Hall, Englewood Cliffs, NJ, 1966.

[22] F. Pereira, J. Sousa, On the approximation of the reachable set boundary, Proc. Controlo 2000. Guimaraes, Portugal 2000, 642-646.

[23] L.S. Pontriagin, The Mathematical Theory of Optimal Processes, Wiley Interscience, New York, 1962.

[24] R. Rodríguez-López, Comparison results for fuzzy differential equations, Information Sciences 178 (2008) 1756-1779.

[25] H. Román-Flores, L. C. Barros and R. C.Bassanezi, A note on Zadeh's extension, Fuzzy Sets and Systems 117 (2001) 327-331.

[26] H. Román-Flores and M.A. Rojas-Medar, Embedding of level-continuous fuzzy sets on Banach spaces, Information Sciences 144 (2002) 227-247.

[27] S. Raczynski, Continuous simulation, differential inclusions, uncertainty and traveling in time, Simulation 80 (2004) 87-100.

[28] T. Rzezuchowski and J. Wasowski, J. Wimagesowski, Differential equations with fuzzy parameters via differential inclusions, Journal of Mathematical Analysis and Applications 255 (2001) 177-194.

[29] R. Vinter, Optimal Control, Birkhäuser, Boston, 2000. 